\title{
Agnus castus fruit extract was safe and effective for relieving symptoms of premenstrual syndrome
}

\author{
Schellenberg $R$, for the study group. Treatment for the premenstrual syndrome with agnus castus fruit extract: prospective, \\ randomised, placebo controlled study. BMJ 2001 Jan 20;322:134-7.
}

\section{QUESTION: Is agnus castus fruit (Vitex agnus castus $L$ extract Ze 440) effective and safe for treatment of premenstrual syndrome?}

\author{
Design \\ Randomised (allocation concealed*), blinded (investiga- \\ tors and patients)*, placebo controlled trial with follow \\ up until the end of the third consecutive menstrual \\ cycle.
}

Setting

6 outpatient general medicine clinics.

\section{Patients}

178 women $\geqslant 18$ years of age who met $D S M-I I I-R$ criteria for premenstrual syndrome. Exclusion criteria were participation in other trials, concomitant psychotherapy, pregnancy or breast feeding, inadequate contraception, dementia, alcohol or drug dependence, serious medical condition, hypersensitivity to agnus castus, fever, pituitary disease, or sex hormone use. 170 women $(96 \%$ follow up) (mean age 36.5 y) were included in the intention to treat analysis.

\section{Intervention}

Women were allocated to receive agnus castus fruit, 20 mg dry tablet once daily $(\mathrm{n}=91)(60 \%$ ethanol $\mathrm{m} / \mathrm{m}$, extract ratio 6-12:1; standardised for casticin), or matching placebo $(n=87)$. Study medication was initiated at the start of the first cycle and continued until the end of the third consecutive cycle.

\section{Main outcome measures}

The main outcome measure was the composite score of the 6 self assessment items (assessed relative to the previous 3 menstrual cycles): irritability, mood alteration, anger, headache, other menstrual symptoms (including bloating), and breast fullness. Items were rated individually using a visual analogue scale (VAS, measured in $\mathrm{mm}$; range 0 [no symptoms] to 10 [unbearable]) and summed for a total score. Secondary outcome measures were responder rate (defined as $\geqslant 50 \%$ improvement in the 6 symptom composite VAS score from baseline), clinical global impression (CGI) items 1 (severity of condition), 2 (improvement or deterioration), and 3 (overall assessment risk or benefit), and adverse events.

\section{Main results}

Women in the agnus castus group had a greater reduction from baseline in the 6 symptom composite VAS score than those in the placebo group (128.5 $v 78.1$, $\mathrm{p}=0.001)$; this group also had a greater responder rate
Agnus castus fruit v placebo in the premenstrual syndrome at the end of 3 consecutive cyclest

\begin{tabular}{lllll} 
Outcome & $\begin{array}{l}\text { Agnus castus } \\
\text { fruit }\end{array}$ & Placebo & RBI (95\% Cl) & NNT (Cl) \\
\hline Responder rate & $52 \%$ & $24 \%$ & $120 \%(45$ to 242$)$ & 4 (2 to 7$)$ \\
\hline
\end{tabular}

(table). Based on individual item VAS scores, women in the agnus castus group had greater reductions in 5 of the 6 symptoms (irritability, mood alteration, anger, headache, and breast fullness; $\mathrm{p} \leqslant 0.002$ ) and showed superiority of the treatment in each of the 3 CGI scores $(\mathrm{p}=0.001)$ than did those in the placebo group. In both groups, adverse events were few $(4.7 \%$ v $4.8 \%$ in agnus castus and placebo groups, respectively) and mild.

\section{Conclusion}

Agnus castus fruit extract was safe and effective for relieving symptoms of premenstrual syndrome.

*See glossary.

\section{COMMENTARY}

This study by Schellenberg shows that a traditional herbal medicine containing sex hormone-like ingredients in an ethanol base has more positive effects on most symptoms of premenstrual syndrome than placebo, and that the beneficial effects last for at least 3 successive menstrual periods.

The study confirms that the differential effect is still there even after the $13 \%$ of women who were taking oral contraceptives were removed from the analysis. The herb was as safe as placebo. In the active group, there was 1 report of urticaria, which may have been an allergic reaction to the herb. The exclusion criterion of "adequate contraception" was shown to be imperfect in that 1 woman in the placebo group had to withdraw from the trial because of pregnancy; pregnancy is always a possible reason for withdrawal in trials involving potentially fertile women. Many patients prefer to take herbs instead of pharmaceuticals (with the belief that herbs might be more "natural"); however, herbs are in fact complex compounds whose active ingredients may be unknown. Agnus castus appears to act as a dopamine agonist, inhibiting prolactin release and probably increasing oestrogen secretion. This herb contains flavonoids that bind to benzodiazepine receptors, and appears to have antidepressant and anxiolytic properties similar to the oestrogen and progesterone hormones. Oestrogen and progesterone hormone concentrations fall during the premenstrual period and the fall is associated with premenstrual dysphoria. Caution must be used in interpreting the results because ethanol was the base used for the herb, and it may be the active ingredient. The follow up period was only 3 months and it is possible that, like other hormones, adverse effects are seen only after chronic use. This study is important because it shows that herbal products contain active ingredients that may be beneficial for certain conditions but that may also have adverse effects, similar to pharmaceuticals. The actual dose of active ingredients, the identity of all ingredients, safety and standards regulations, indications, and contraindications all need to be specified to the same extent as currently seen for pharmaceutical products.

Mary V Seeman, MD Centre for Addiction and Mental Health Toronto, Ontario, Canada 